\title{
Interplay of serum hepcidin with female sex hormones, metabolic syndrome, and abdominal fat distribution among premenopausal and postmenopausal women
}

\author{
Ragaa Abdelshaheed Matta ${ }^{1 *} \mathbb{D}$, Mohamed Emad AbdElftah², Mohamed Gaber Essawy ${ }^{3}$ and
} Ahmed Abdelfadel Saedii ${ }^{4}$

\begin{abstract}
Background and purpose: Hepcidin is the central regulatory molecule of systemic iron homeostasis. Serum ferritin, insulin resistance (IR) and metabolic syndrome (MetS), female sex hormones, and abdominal fat distribution are related to each other and all are linked to menopausal state. Our study was the first to assess the impact of these parameters on hepcidin level among premenopausal women (group I) during the early follicular phase (group I-F) and mid-luteal-phase (group I-L) of the same reproductive cycle and among postmenopausal women (group II). Serum iron parameters, estrogen, progesterone and hepcidin, and plasma insulin were assessed. Abdominal subcutaneous fat (SCF) and peritoneal visceral fat (PVF) thickness were measured by unenhanced- CT. Group I and group II were divided into MetS and non-MetS subgroups.

Results: The entire group II and MetS-stratified subgroups had significant higher hepcidin level than corresponding group I-F and group I-L. Group I-L had significant higher hepcidin than group I-F. Among group I-F, group I-L, and group II, MetS subgroups had higher hepcidin but not hepcidin/ ferritin ratio (H/F) than corresponding non-MetS; and hepcidin had positive correlations with ferritin, insulin, IR, and SCF. In group I-F and group II, hepcidin had positive correlations with estrogen and progesterone; hepcidin levels increase significantly and linearly with increasing number of MetS features; and cut off values of hepcidin for prediction of MetS were $5.8 \geq$ and $\geq 10.3 \mathrm{ng} / \mathrm{ml}$ respectively. Main contributors to hepcidin were iron and ferritin in all groups, SCF and progesterone in group I-F, and insulin, progesterone, and MetS in group II. H/F ratio was higher in group II.

Conclusion: Postmenopausal state (postMS), MetS, and luteal phase are independently associated with high hepcidin level. Serum iron parameters (iron and ferritin) as main regulators of hepcidin are preserved regardless of menopausal state. Its regulation differs based on menopausal state: IR, MetS, and progesterone in postMS meanwhile abdominal SCF and progesterone in premenopausal states. Despite positive associations of estrogen and progesterone with hepcidin, they do not explain its higher level in postMS. Hepcidin levels linearly increase with number of Mets feature and it had high sensitivity for diagnosis of MetS.
\end{abstract}

\footnotetext{
*Correspondence: ragaamatta2017@gmail.com

${ }^{1}$ Diabetes and Endocrinology Unit, Department of Internal Medicine,

Faculty of Medicine, Minia University, Minia, Egypt

Full list of author information is available at the end of the article
} original author(s) and the source, provide a link to the Creative Commons licence, and indicate if changes were made. The images or other third party material in this article are included in the article's Creative Commons licence, unless indicated otherwise in a credit line to the material. If material is not included in the article's Creative Commons licence and your intended use is not permitted by statutory regulation or exceeds the permitted use, you will need to obtain permission directly from the copyright holder. To view a copy of this licence, visit http://creativecommons.org/licenses/by/4.0/. 


\section{Highlights}

- Postmenopausal state (PMS) and metabolic syndrome (MetS) are independent factors of high hepcidin.

- Estrogen and progesterone are correlated with hepcidin but not explain its higher level in PMS.

- Ferritin, insulin resistance, and MetS are main contributors of high hepcidin in PMS.

- High hepcidin in luteal phase is attributed to ferritin and abdominal subcutaneous fat

- Hepcidin levels linearly increase with number of Mets feature and it had high sensitivity for diagnosis of MetS

Keywords: Postmenopausal state, Reproductive cycle phase, Hepcidin, Abdominal fat, Metabolic resistance, Female sex hormones

\section{Background}

Hepcidin as the central regulatory molecule of systemic iron homeostasis is secreted predominantly by the hepatocytes and to lesser extent by the adipose tissue. Hepcidin controls cellular iron efflux via binding to the cellular iron exporter ferroportin and inducing its internalization and degradation. Elevated hepcidin levels lead to decrease dietary iron absorption and prevent the release of iron from hepatocytes and macrophages, resulting in hypoferremia and elevated serum ferritin [1].

Clinical, biochemical, metabolic, and hormonal changes physiologically occur over female reproductive cycle and with the development of menopause. Insulin level starts to increase just before ovulation and reaches the highest level during luteal phase. This was attributed to estradiol and progesterone levels [2]. The serum iron and hepcidin fall during early follicular phase (EFP) then rebound rise and establish during luteal phase of menstrual cycle [3]. In addition, the postmenopausal state (postMS) is associated with significant higher ferritin, insulin resistance (IR), metabolic syndrome (MetS) prevalence, and abdominal fat and significant lower both estradiol and progesterone levels than premenopausal state (preMS). Based on age as a proxy in the absence of data on menopausal state, hepcidin was substantially higher in postMS than the preMS. Of note, postMS is considered as a risk factor for MetS development [4-7]. Estrogen and progesterone may regulate hipcidin gene expression via binding to their specific response elements. The role of estrogen in enhancement or suppression of hepcidin expression is still unclear as both elevated and decreased hepatic hepcidin transcription were described in ovariectomized mice and cellular studies [8-10]. Recent in vitro fertilization study demonstrated that reduced circulating hepcidin was concomitant with enhanced endogenous estrogen production by 5-folds [11]. On the other hand, progesterone enhances hipcidin synthesis in experimental animals, cell culture, and among non-pregnant women undergoing in vitro fertilization [12].
Association of IR with elevated ferritin is bidirectional and is known as dysmetabolic hyperferritinemia (DH) which is illustrated by elevated ferritin and features of MetS. Hepcidin/ferritin ratio (H/F), reflecting hepcidin expression relative to iron stores may clinically help for discrimination of DH from other iron overload condition as hereditary hemochromatosis where hepcidin is inappropriately low. Elevated H/F may signify impaired hepcidin action or may indicate other factors which rise hepcidin level independent of serum ferritin such as inflammatory cytokines. So, hepcidin resistance may underly DH pathogenesis as hepcidin seems to play a role in iron accumulation as the iron challenge does not restrain iron absorption despite adequate hepcidin production [13-15]. Ferritin is the main regulator for hipcidin level in male and females; meanwhile, MetS was a contributor to higher hepcidin level only in females in a population study [16]. However, another study demonstrated the association of hepcidin, ferritin, and MetS most frequently in men [17]. Associations of serum hepcidin with insulin level, homeostatic model for assessment of insulin resistance (HOMA-IR), and IR conditions reveal marked variability in different population studies [1820]. The contribution of adipose tissue derived hepcidin to the circulating hepcidin levels is still unclear. Researchers suggested major role of adipose tissue in elevated hepcidin among obese premenopausal women while others reported actually very little contribution of adipose tissue [21, 22].

Up to date, roles of MetS, female sex hormones, IR, abdominal fat distribution as factors that could potentially have a great effect in determining the hipcidin in women based on menopausal state were not addressed before. The aim of this study is to explore the possible influence and interplay of female sex hormones and IR and MetS, abdominal fat on hepcidin level in the preMS during the EFP and mid luteal phase (MLP) and in the postMS. 


\section{Subjects and method}

The present observational case-control study included 160 women who were divided according to menopausal state into preMS group (group I, $n=96$ ) and in postMS group (group II, $n=64$ ). preMS group was assessed during EFP and MLP of same menstrual cycle and termed group I-F and group I-L respectively. Both the preMS and postMS groups were further subdivided into metabolic and non- metabolic subgroups (with metabolic/ non-metabolic ratios of $45 / 51$ and $36 / 28$, respectively). The present study was conducted in Endocrinology and Diabetic unit, Internal Medicine Department from June 2016 to June 2017. The subjects were selected from attendant to outpatient clinic for checkup examination. Premenopausal women had regular menstrual cycle (21-35 days) and menses length between 3 and 5 days. Menopause was defined as permanent cessation of menstrual periods, determined retrospectively after a woman has experienced 12 months of amenorrhea without any obvious pathological or physiological causes [23]. According to established criteria, MetS diagnosis was based on the presence of at least three of the following five features: waist circumference $\geq 88 \mathrm{~cm}$; fasting plasma glucose (FPG) $\geq 100 \mathrm{mg} / \mathrm{dL}$; serum triglycerides $\geq 150 \mathrm{mg} /$ $\mathrm{dL}$; serum high-density lipoprotein-cholesterol (HDL-C) $\leq 50 \mathrm{mg} / \mathrm{dL}$; and blood pressure $\geq 130 / 85 \mathrm{mmHg}$ [24]. Women that met any one of the following exclusion criteria were not included in the study: with age $<20$ years, pregnancy, lactation, or irregular menses, anemia, recent infection or inflammation, cardiovascular disorder, diabetes mellitus, chronic illness, organ dysfunction, and malignancy anywhere or taking medications or hormonal therapy that may influence the studied laboratory parameters or previous ovarian surgery.

The study protocol was approved by the local Institutional Ethics Committee and was conducted in accordance with Declaration of Helsinki. All the subjects gave written informed consent before enrolling in the study.

Through history taking and anthropometric measurements that included waist circumference and systemic arterial blood pressure were assessed according to standard methods.

\section{Biochemical and hormonal investigations}

For each premenopausal woman, the laboratory investigations were carried out over one menstrual cycle when blood samples were collected twice; one during EFP (3-5 days) and another in MLP (18-22 days). Blood samples were taken once from postmenopausal females. An overnight fasting and 12-h fasting blood samples were collected at 9:00 AM and prepared for routine laboratory investigations including the measurement of FPG and complete lipogram according to standard laboratory methods. The stored serum and plasma samples at -70 ${ }^{\circ} \mathrm{C}$ were used for the following analysis according to recommendations of the kit's manufacturers. Serum iron was determinate via quantitative colorimetric method using kits supplied by STANBIO laboratory, USA. Quantitative determination of plasma insulin and serum ferritin, estradiol, and progesterone concentration was done via ELISA kits (Chemux BioScience Inc, South San Francisco, USA). Serum hepcidin was assessed via sandwich-ELISA (Elabscience Biotechnology Co., Ltd, China): HOMA- IR was calculated [25].

\section{Measurement of abdominal fat}

Unenhanced computerized tomography (CT) scan images were obtained to measure peritoneal visceral fat thickness (PVF) and subcutaneous fat thickness (SCF) at the level of the top of the iliac crest.

\section{Statistical analysis}

In all the statistical analyses, the SPSS software version 22.0 was used. Data for continuous normally were expressed as mean \pm SD and analyzed using the Student's $t$ test or by analysis of variance (ANOVA) with polynomial contrasts for linear trend, when appropriate. Data for continuous non-normally distributed data were expressed as median (interquartile range) and compared using the Mann-Whitney test. Qualitative data were analyzed with the chi-squared test and with chi-squared analysis for linear trend, when appropriate. Paired sample $t$ test was used to compare EFP and MLP of the reproductive cycle in the premenopausal group. Correlations between quantitative variables were assessed using Spearman's or Pearson's correlation. Independent predictors of serum hepcidin levels were assessed through stepwise linear regression analysis. Receiver operating characteristic (ROC) curve analysis was performed to evaluate the diagnostic utility of hepcidin for diagnosis of MetS. Areas under curves (AUCs) were measured to calculate specificity and sensitivity. A $p$ value $<0.05$ was considered statistically significant.

\section{Results}

Premenopausal and postmenopausal women were matched as regard proportion of MetS ( $46 \%$ vs. $56 \%, p=$ 0.26).

Table 1 summarizes the main clinical, anthropometric and biochemical features, and abdominal fat distribution among the studied groups and subgroups. Compared to preMS, postMS was associated with significant higher systolic blood pressure, diastolic blood pressure, FPG, and abdominal SCF among entire subjects and stratified MetS and non-MetS subgroups. We also found significant higher (low-density lipoprotein-cholesterol) LDL-.c, 
Table 1 Baseline characteristics, biochemical and abdominal fat of premenopausal, and postmenopausal women stratified for having or not the metabolic syndrome

\begin{tabular}{|c|c|c|c|c|c|c|}
\hline \multirow[t]{2}{*}{ Variables } & \multicolumn{3}{|c|}{ Premenopausal women } & \multicolumn{3}{|c|}{ Postmenopausal women } \\
\hline & Total $(n=96)$ & Non- MetS $(n=51)$ & MetS $(n=45)$ & Total $(n=64)$ & Non-MetS $(n=28)$ & MetS $(n=36)$ \\
\hline Age (years) & $32.5 \pm 5.9$ & $32.5 \pm 6.5$ & $32.5 \pm 5.1$ & $57.1 \pm 6.6^{* * *}$ & $56.9 \pm 6.2^{* * *}$ & $57.2 \pm 6.8^{* * *}$ \\
\hline Waist cir (cm) & $102.7 \pm 11$ & $95.8 \pm 9.5$ & $110.4 \pm 6.2$ & $105.5 \pm 10.5$ & $97.8 \pm 7.2$ & $111.4 \pm 8.7$ \\
\hline $\mathrm{SBP}(\mathrm{mmHg})$ & $123 \pm 9.6$ & $115.8 \pm 5.8$ & $131 . \pm 6.1$ & $128.4 \pm 11^{* *}$ & $118.9 \pm 7.4^{*}$ & $135.7 \pm 6.7^{* *}$ \\
\hline $\mathrm{DBP}(\mathrm{mmHg})$ & $74.9 \pm 7.5$ & $70.2 \pm 5.6$ & $80.0 \pm 5.9$ & $78.5 \pm 6.1^{* *}$ & $73.5 \pm 4.48^{* *}$ & $82.2 \pm 4.26^{*}$ \\
\hline $\mathrm{HB} \%(\mathrm{~g} / \mathrm{dl})$ & $12.7 \pm 0.5$ & $12.5 \pm 0.43$ & $12.48 \pm 0.43$ & $12.8 \pm 0.8$ & $12.7 \pm 0.48$ & $13 . \pm 0.93$ \\
\hline FBS (mg/dl) & $89.8 \pm 15.5$ & $82.4 \pm 9.2$ & $98.1 \pm 17$ & $100 \pm 15^{* * *}$ & $88.1 \pm 11.2^{*}$ & $109 \pm 11^{* *}$ \\
\hline $\mathrm{TC}(\mathrm{mg} / \mathrm{dl})$ & $203.5 \pm 54.7$ & $170.2 \pm 47.8$ & $241.2 \pm 33.5$ & $200.6 \pm 47.6$ & $168.7 \pm 34.4$ & $225.3 \pm 41.3$ \\
\hline $\mathrm{TG}(\mathrm{mg} / \mathrm{dl})$ & $104.5 \pm 31.9$ & $86.7 \pm 25.0$ & $124.6 \pm 26.6$ & $113.1 \pm 41.1$ & $81.0 \pm 21.6$ & $138.0 \pm 34.9$ \\
\hline LDL-C (mg/dl) & $144.7 \pm 47$ & $115.7 \pm 37.8$ & $177.4 \pm 33$ & $136.1 \pm 46.6$ & $109.4 \pm 32$ & $156.8 \pm 45.7^{*}$ \\
\hline $\mathrm{HDL}-\mathrm{C}(\mathrm{mg} / \mathrm{dl})$ & $44.9 \pm 9.5$ & $50.2 \pm 8.4$ & $38.7 \pm 6.4$ & $46.8 \pm 11.3$ & $51.4 \pm 11.1$ & $43.2 \pm 10.1^{*}$ \\
\hline $\mathrm{SCF}(\mathrm{cm})$ & $3 \pm 0.7$ & $2.69 \pm 0.85$ & $3.4 \pm 0.5$ & $3.5 \pm 0.6^{* * *}$ & $3.06 \pm 0.14^{*}$ & $3.8 \pm 0.4^{* *}$ \\
\hline $\mathrm{PVF}(\mathrm{cm})$ & $5.4 \pm 0.9$ & $5.1 \pm 1.02$ & $5.7 \pm 1.09$ & $5.8 \pm 1.2^{* *}$ & $4.78 \pm 0.5$ & $6.2 \pm 1.2^{*}$ \\
\hline
\end{tabular}

Quantitative data are expressed as Mean \pm SD and compared by using independent samples $T$ test. Compared to corresponding premenopausal women, significant level: $p<0.05={ }^{*}, p<0.01={ }^{* *}, p<0.001^{* * *}$

MetS Metabolic syndrome, Waist cir Waist circumference, SBP Systolic blood pressure, DBP Diastolic blood pressure, HB Hemoglobin, FBS Fasting blood sugar, TC Total cholesterol, TG Triglycerides, LDL-C Low-density lipoprotein-cholesterol, HDL-C High-density lipoprotein-cholesterol, SCF Subcutaneous fat thickness, PVF Peritoneal visceral fat thickness

HDL-c, and PVF only in metabolic subgroups and significant higher PVF among entire subjects of postMS than corresponding preMS.

Table 2 shows the iron and hormonal parameters during EFP and MLP in preMS and in postMS. Among total subjects and stratified metabolic and non-metabolic subgroups: The statistical significant ascending order for the studied phases and states according to serum iron parameters (iron, ferritin, hepcidin, $\mathrm{H} / \mathrm{P}$ ratio) was EFP, MLP, and postMS respectively except for $\mathrm{H} / \mathrm{F}$ ratio when MLP was compared to either postMS in non- metabolic subgroups or EFP in metabolic subgroups. Moreover, the statistical significant ascending order according to serum estradiol and progesterone levels was postMS, EFP, and MLP except for progesterone when EFP was compared to postMS in non-metabolic groups. Plasma insulin and HOMA-IR were significantly lower in the EFP than in both MLP and postMS along with insignificant difference between the latter conditions among entire subjects and stratified metabolic groups and between the EFP and postMS in non-metabolic groups. MLP was associated with higher insulin and IR than postMS in non -metabolic subgroups (Table 2).

\section{Comparison of metabolic and non-metabolic subgroups within the same phase or state}

Among EFP, MLP, and postMS, metabolic groups had significant lower iron and significant higher ferritin, hepcidin, insulin, and HOMA-IR than non-metabolic groups. Metabolic group of EFP showed higher estradiol and progesterone and metabolic group of postMS had higher estradiol than phase or state matched non- metabolic group (Table 2).

\section{Association of metabolic syndrome and hepcidin level}

We stratified hepcidin levels into quartiles to evaluate the proportion of MetS with each quartile. Among both preMS and postMS, MetS\% significantly rise with ascending order's hepcidin quartiles (Table 3(A)). Also, serum hepcidin levels were significantly increased according to increasing number of MetS features (Table 3(B)).

ROC curve analysis was done to evaluate the diagnostic ability of hepcidin for MetS. Cut off value of hepcidin for diagnosis of MetS was $\geq 5.8 \mathrm{ng} / \mathrm{ml}$ and $\geq 10.3 \mathrm{ng} / \mathrm{ml}$ in preMS and postmMS respectively. The AUC, the sensitivity, and specificity were summarized in Table $3(\mathrm{C})$ and Figs. 1 and 2.

\section{Bivariate and multivariate correlations for hepcidin}

\section{Correlations of hepcidin with component of MetS}

Hepcidin had significant positive correlations and waist circumference, SBP, DBP, triglyceride, FPG, and significant negative correlations with HDL-c in preMS and postMS $(p<0.001$ for all).

2. Serum hepcidin had significant negative correlation with iron and significant positive correlations 
Table 2 Iron and insulin parameters of the luteal, follicular phases of menstrual cycle and postmenopausal state stratified of having or not the MetS

\begin{tabular}{|c|c|c|c|c|c|c|c|}
\hline & \multirow[t]{2}{*}{ MetS } & \multicolumn{2}{|c|}{ Premenopausal women } & \multirow{2}{*}{$\begin{array}{l}\text { Post menopause } \\
\text { women (PMS) }\end{array}$} & \multicolumn{3}{|l|}{$P$ value } \\
\hline & & EFP & MLP & & EFP vs. MLP & EFP vs. PMS & MPI vs. PMS \\
\hline \multirow[t]{4}{*}{ Iron $(\mu \mathrm{g} / \mathrm{dl}) \mathrm{a}$} & Total & $43 \pm 8.4$ & $84.3 \pm 18$ & $126.8 \pm 33$ & $<0.001$ & $<0.001$ & $<0.001$ \\
\hline & Yes & $41 \pm 9.9$ & $79.3 \pm 15.6$ & $114.8 \pm 30.9$ & $<0.001$ & $<0.001$ & $<0.001$ \\
\hline & No & $44.7 \pm 6.5$ & $88.5 \pm 19.0$ & $142.4 \pm 29.4$ & $<0.001$ & $<0.001$ & $<0.001$ \\
\hline & P1 & 0.035 & 0.01 & 0.001 & & & \\
\hline \multirow[t]{4}{*}{ Ferritin (ng/ml) a } & Total & $67.6 \pm 24.3$ & $92 \pm 35.5$ & $118.6 \pm 44.7$ & $<0.001$ & $<0.001$ & $<0.001$ \\
\hline & Yes & $84.9 \pm 16.1$ & $119 \pm 21.9$ & $139.8 \pm 43.3$ & $<0.001$ & $<0.001$ & 0.007 \\
\hline & No & $52.4 \pm 19.7$ & $67.8 \pm 26.4$ & $91.2 \pm 29.1$ & $<0.001$ & $<0.001$ & $<0.001$ \\
\hline & P1 & $<0.001$ & $<0.001$ & $<0.001$ & & & \\
\hline \multirow[t]{4}{*}{ Hepcidin (ng/ml) a } & Total & $6.5 \pm 2.6$ & $9.5 \pm 3.7$ & $12.8 \pm 3.8$ & $<0.001$ & $<0.001$ & $<0.001$ \\
\hline & Yes & $8.4 \pm 1.9$ & $12.2 \pm 2.3$ & $15 \pm 3$ & $<0.001$ & $<0.001$ & $<0.001$ \\
\hline & No & $4.9 \pm 1.9$ & $7.06 \pm 2.8$ & $9.8 \pm 2.6$ & $<0.001$ & $<0.001$ & $<0.001$ \\
\hline & P1 & $<0.001$ & $<0.001$ & $<0.001$ & & & \\
\hline \multirow[t]{4}{*}{$\mathrm{H} / \mathrm{F}$ ratio a } & Total & $0.09 \pm 0.02$ & $0.1 \pm 0.02$ & $0.113 \pm 0.03$ & $<0.001$ & $<0.001$ & 0.02 \\
\hline & Yes & $0.09 \pm 0.01$ & $0.10 \pm 0.01$ & $0.11 \pm 0.03$ & 0.14 & 0.005 & 0.03 \\
\hline & No & $0.09 \pm 0.02$ & $0.10 \pm 0.01$ & $0.11 \pm 0.03$ & 0.03 & 0.01 & 0.14 \\
\hline & P1 & 0.490 & 0.84 & 0.946 & & & \\
\hline \multirow[t]{4}{*}{ Insulin $(\mathrm{Mu} / \mathrm{ml}) \mathrm{b}$} & Total & $11.5[9-16]$ & 14 [12-19] & 14 [6.3-19] & $<0.001$ & 0.04 & 0.249 \\
\hline & Yes & 16 [11.-18] & 19 [15-25] & 18 [15-26] & $<0.001$ & 0.03 & 0.86 \\
\hline & No & $9.0[7-12]$ & $12[10-14]$ & $8.5[4-11]$ & $<0.001$ & 0.17 & $<0.001$ \\
\hline & P1 & $<0.001$ & $<0.001$ & $<0.001$ & & & \\
\hline \multirow[t]{4}{*}{ HOMA-IR b } & Total & $1.55[1.1-2.1]$ & $1.8[1.6-2.57]$ & $1.9[1.05-2.8]$ & $<0.001$ & 0.04 & 0.385 \\
\hline & Yes & $2.0[1.5-2.3]$ & $2.5[1.9-3.2]$ & $3.5[2.45-4.6]$ & $<0.001$ & 0.005 & 0.88 \\
\hline & No & $1.1[0.9-1.6]$ & $1.6[1.3-1.8)$ & $1.4[1.1-2.1]$ & $<0.001$ & 0.16 & $<0.001$ \\
\hline & P1 & $<0.001$ & $<0.001$ & $<0.001$ & & & \\
\hline \multirow[t]{4}{*}{ Estradiol (pg/ml) a } & & $53.4 \pm 16.8$ & $91.9 \pm 26.4$ & $26.1 \pm 12.9$ & $<0.001$ & $<0.001$ & $<0.001$ \\
\hline & Yes & $57.4 \pm 16.2$ & $93.6 \pm 22.0$ & $31.3 \pm 13.5$ & $<0.001$ & $<0.001$ & $<0.001$ \\
\hline & No & $49.9 \pm 16.8$ & $90.2 \pm 29.8$ & $19.4 \pm 8.2$ & $<0.001$ & $<0.001$ & $<0.001$ \\
\hline & P1 & 0.030 & 0.53 & $<0.001$ & & & \\
\hline \multirow[t]{4}{*}{ Progesterone $(\mathrm{ng} / \mathrm{ml}) \mathrm{b}$} & & $1.4[0.96-1.9]$ & $10.5[4.3-13.7]$ & $1.1[0.5-1.5]$ & $<0.001$ & $<0.001$ & $<0.001$ \\
\hline & Yes & $1.7[1.3-2.7]$ & $12.2[5.3-16.7]$ & $1.1[0.45-1.5]$ & $<0.001$ & $<0.001$ & $<0.001$ \\
\hline & No & $1.29[0.9-1.5]$ & $7.5[3.5-12.9]$ & $1.1[0.68-1.3]$ & $<0.001$ & 0.18 & $<0.001$ \\
\hline & P1 & $<0.001$ & 0.20 & 0.49 & & & \\
\hline
\end{tabular}

Parametric and non- parametric quantitative data are expressed as Mean \pm SD and median and 25-75\% interquartile and compared using student $t$ test and Mann-Whitney between EFP and MLP vs. PMS and using paired samples $T$ and Wilcoxon signed rank test between EFP vs. MLP respectively. P1 $=p$-value when we compared metabolic versus non-metabolic within the same phase or state. Significant level is at $p$ value $<0.05$ and written in bold

MetS Metabolic syndrome, EFP Early follicular phase, MLP Mid-luteal phase, PMS Postmenopausal state, H/F Hepcidin/ferritin, HOMA-IR Homeostatic model assessment of insulin resistance

with ferritin, insulin and HOMA-IR, abdominal SCF, MetS features' number in EFP, MLP, and postMS. Hepcidin had positive correlations with estradiol and progesterone in EFP and postMS. Hepcidin was positively correlated with H/F ratio in EFP and with abdominal PVF in postMS. During reproductive cycle, percent increase of serum hepcidin (MLP-EFP/ EFP) was inversely correlated to corresponding per- centage increased estradiol, insulin, and HOMA-IR and positively correlated to $\mathrm{H} / \mathrm{F}$ ratio $(p<0.001)$.

3. By doing multivariate analysis with hepcidin as the dependent factor, the independent factors of hepcidin were iron ferritin, progesterone, and abdominal SCF in EFP, serum iron, ferritin and SCF in MLP, and iron, ferritin, progesterone, insulin, and MetS in postMS (Table 4). 
Table 3 Association of serum hepcidin and metabolic syndrome (MetS)

A-Association of MetS \% and hepcidin quartiles (Qs)

Premenopausal

$\begin{array}{lllll}\text { Hepcidin Qs } & \text { Q1 }(n=24) & \text { Q2 }(n=24) & \text { Q3 }(n=24) & \text { Q4 }(n=24) \\ \text { MetSn (\%) } & \text { O(0\%) } & 9(37.5 \%) & 12(50 \%) & \text { 24(100\%) } \\ \text { Pvalue } & <\mathbf{0 . 0 0 1} & & & \end{array}$

Pvalue

$<0.001$

B-Association of serum hepcidin ( $\mathrm{ng} / \mathrm{ml}$ ) with MetS features' number

MetSfeatures (n) Premenopausal

$\begin{array}{llllll} & \mathbf{0} & \mathbf{1} & \mathbf{2} & \mathbf{3} & \mathbf{4} \\ \text { Subjects }(n) & 12 & 6 & 33 & 27 & 18 \\ \text { Hepcidin level } & 3.2 \pm 0.6 & 4.0 \pm 0.7 & 5.6 \pm 1.8 & 7.8 \pm 1.9 & 9.4 \pm 1.6 \\ \text { Pvalue } & <\mathbf{0 . 0 0 1} & & & & \\ \text { Croc } & & & & \end{array}$

Post-menopause

\begin{tabular}{|c|c|c|c|}
\hline $\mathrm{Q} 1(n=16)$ & $\mathrm{Q} 2(n=16)$ & $\mathrm{Q} 3(n=16)$ & $\mathrm{Q} 4(n=16)$ \\
\hline $0(0 \%)$ & $10(62.5 \%)$ & $10(62.5 \%)$ & $16(100 \%)$ \\
\hline
\end{tabular}

$<0.001$

\section{C-ROC curve analysis of hepcidin $(\mathrm{ng} / \mathrm{ml})$ for prediction of metabolic syndrome}

\begin{tabular}{llllllll} 
& Optimal cutoff & AUC & Pvalue & Sensitivity & Specificity & PPV & Accuracy \\
Premenopausal & $\geq 5.8$ & 0.910 & $<\mathbf{0 . 0 0 1}$ & 93.3 & 76.5 & $\mathbf{7 7 . 8}$ & $\mathbf{8 4}$ \\
Postmenopausal & $\geq 10.3$ & 0.907 & $\mathbf{< 0 . 0 0 1}$ & 100 & 71.4 & 81.8 & $\mathbf{8 0}$ \\
\hline
\end{tabular}

Bold: significant at $p$ value $<0.05$

AUC Area under the curve, PPV Positive predictive value, NPV Negative predictive value

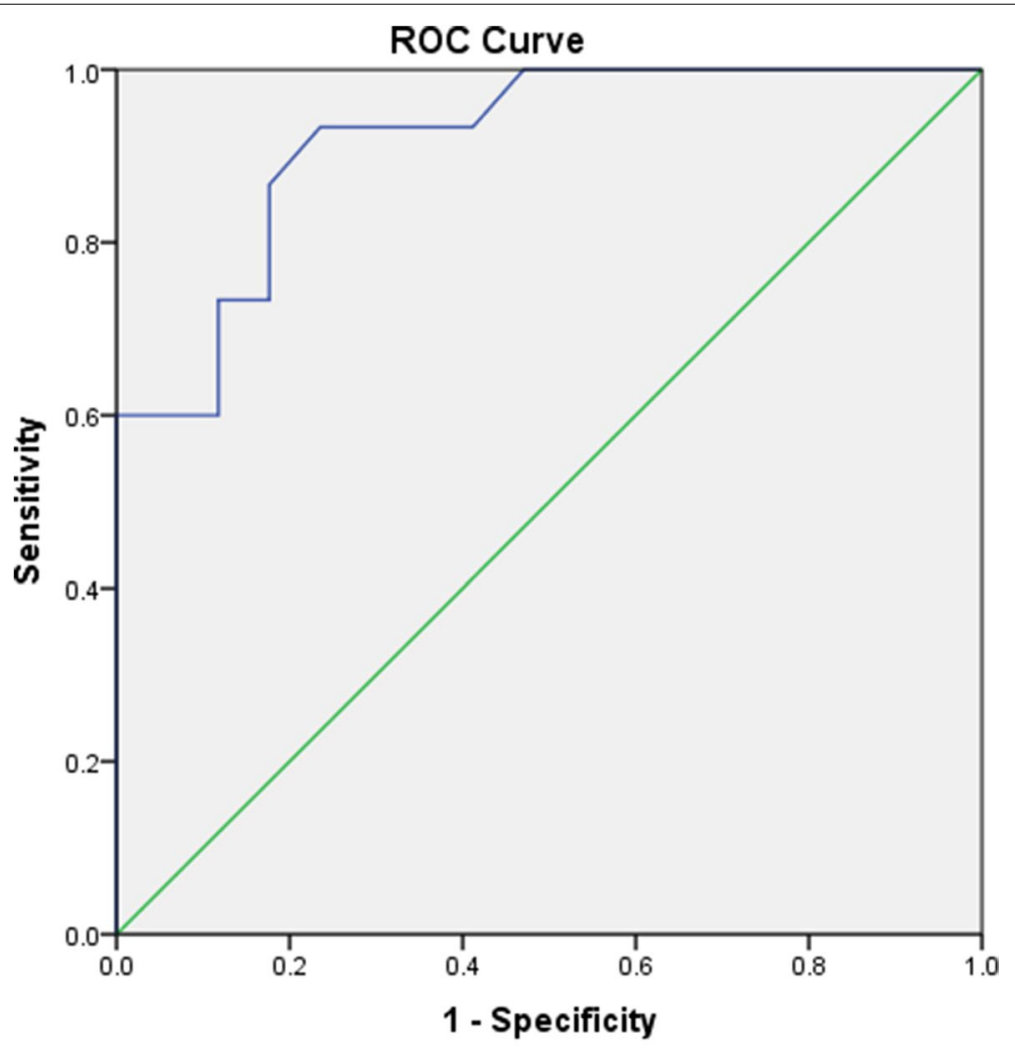

Diagonal segments are produced by ties.

Fig. 1 ROC curve for prediction of metabolic syndrome in premenopausal women. ROC curve for prediction of metabolic syndrome in premenopausal women using cutoff value of $\geq 5.8 \mathrm{ng} / \mathrm{ml}$ for hepcidin. The AUC, sensitivity, and specificity were $0.910,93.3 \%, 76.5 \%$ respectively with $p$ values $\leq 0.001$ 


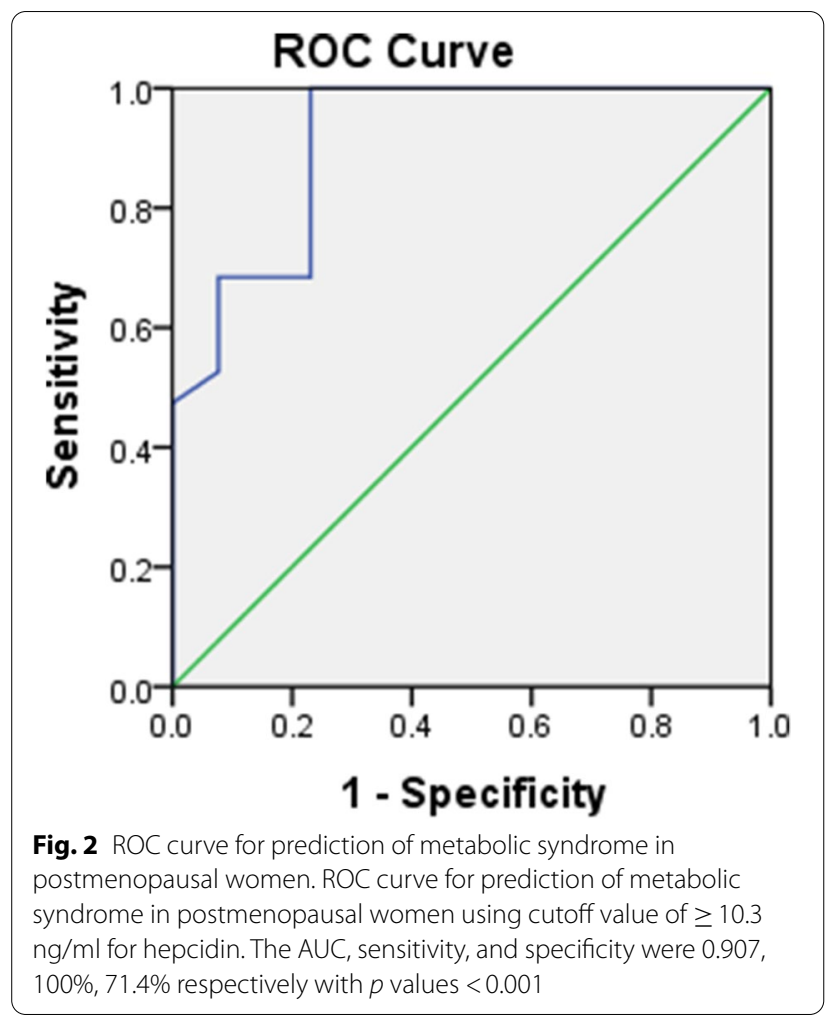

\section{Discussion}

Our study had a novelty to address the roles of female sex hormones, MetS, IR, and abdominal fat distribution in addition to preserved defined role of serum ferritin for elevated hepcidin level in post-MS and during MLP of reproductive cycle in premenopausal women.

We reported higher hipcidin level in postMS than preMS and in MLP than EFP of reproductive cycle regardless of the MetS. These findings were matched to previous population and Hepcidin During Menses (HEPMEN) studies [3, 5]. Fortunately, our study took precedence in accurate definition of menopause that depended on menses caseation while previous population study used age only as a proxy. HEPMEN study demonstrated initial reduction of both hipcidin and the iron during menstrual phase followed by their rebound rise and steady state in the luteal phase of the cycle. That was similar to our data but we did not agree with their state of unremarkable variation of serum ferritin during the reproductive cycle [3].Of note, $61 \%$ of women in HEPMEN study were on oral contraceptives which represent a limitation of their study as hormonal contraceptive increase iron and ferritin and subsequently it may interfere with interpretation of the results [26]. Our study overcame this limitation as intake of hormonal contraception was an exclusion criterion. We also were pioneering in reporting elevated hepcidin levels in postMS and MLP among those with and without MetS.

Association of hepcidin with menopausal state and reproductive cycle phases encouraged us to study its association with female sex hormones. Although estradiol and progesterone were positively correlated with hepcidin level during EFP in PreMS and in postMS in our study, only progesterone was independent predictor of hipcidin in multivariate analysis. Previous data

Table 4 Correlations of hepcidin and linear regression analysis for hepcidin as dependent variable

\begin{tabular}{|c|c|c|c|c|c|c|c|c|c|c|c|c|}
\hline & \multicolumn{4}{|c|}{ Early follicular phase } & \multicolumn{4}{|c|}{ Mid Luteal phase } & \multicolumn{4}{|c|}{ Post-menopause } \\
\hline & \multicolumn{2}{|c|}{ Bivariate } & \multirow{2}{*}{\multicolumn{2}{|c|}{$\begin{array}{l}\text { Multivariate } \\
R=0.89 \\
\text { Adjact } R^{2}=0.78\end{array}$}} & \multicolumn{2}{|c|}{ Bivariate } & \multirow{2}{*}{\multicolumn{2}{|c|}{$\begin{array}{l}\text { Multivariate } \\
R=0.83 \\
\text { Adjact } \mathrm{R}^{2}=0.68\end{array}$}} & \multicolumn{2}{|c|}{ Bivariate } & \multirow{2}{*}{\multicolumn{2}{|c|}{$\begin{array}{l}\text { Multivariate } \\
R=0.93 \\
\text { Adjact } \mathrm{R}^{2}=0.85\end{array}$}} \\
\hline & \multirow[b]{2}{*}{$\mathbf{r}$} & \multirow[b]{2}{*}{$p$-value } & & & & & & & & & & \\
\hline & & & Beta & $p$-value & $r$ & $p$-value & Beta & $p$-value & $r$ & $p$-value & Beta & $p$-value \\
\hline & & & Cons & 0.22 & & & Cons & 0.147 & 0.64 & $<0.001$ & Cons & $<0.001$ \\
\hline Iron & -0.38 & $<0.001$ & 0.20 & $<0.001$ & -0.57 & $<0.001$ & -0.08 & 0.02 & -0.57 & $<0.001$ & 0.15 & 0.005 \\
\hline Ferritin & 0.82 & $<0.001$ & 0.51 & $<0.001$ & 0.95 & $<0.001$ & 0.84 & $<0.001$ & 0.77 & $<0.001$ & 0.48 & $<0.001$ \\
\hline H/F ratio & 0.39 & $<0.001$ & & & 0.143 & 0.165 & & & 0.02 & 0.853 & & \\
\hline Estradiol & 0.25 & 0.007 & & & 0.19 & 0.06 & & & 0.51 & $<0.001$ & & \\
\hline Progesterone & 0.45 & $<0.001$ & 0.22 & $<0.001$ & -0.02 & 0.81 & & & 0.36 & 0.003 & -0.20 & 0.002 \\
\hline Insulin & 0.55 & $<0.001$ & & & 0.77 & $<0.001$ & & & 0.78 & $<0.001$ & 0.24 & 0.001 \\
\hline HOMA-IR & 0.55 & $<0.001$ & & & 0.75 & $<0.001$ & & & 0.78 & $<0.001$ & & \\
\hline $\mathrm{SCT}$ & 0.62 & $<0.001$ & 0.31 & $<0.001$ & 0.62 & $<0.001$ & 0.1 & 0.004 & 0.67 & $<0.001$ & & \\
\hline PVF & 0.17 & 0.96 & & & 0.17 & 0.96 & & & 0.49 & $<0.001$ & & \\
\hline MetS & 0.79 & $<0.001$ & & & 0.75 & $<0.001$ & & & 0.83 & $<0.001$ & 0.18 & 0.006 \\
\hline
\end{tabular}

H/F Ratio hepcidin/ferritin ratio, HOMA-IR Homeostatic model assessment of insulin resistance, SCF Subcutaneous fat thickness, PVF Peritoneal visceral fat thickness, PVF, MetS Metabolic syndrome 
described these associations is still limited and unclear as stimulatory and inhibitory effect of estradiol on hepatic hepcidin expression via a functional estradiol responsive element within its promoter were reported. In addition, both downregulation and unchanged hepcidin expression were described in ovariectomized mice $[8-10,12]$. In light of the clinical study among healthy females, suppression and stimulation of endogenous estrogen using gonadotropin-stimulating hormone agonist and folliclestimulating hormone injection respectively as a part of in-vitro fertilization led to in 5-fold rise of estrogen during stimulation phase and concomitant $40 \%$ reduction of hipcidin level without change of serum iron or ferritin [11]. We reported $\uparrow \Delta \%$ estradiol was inversely correlated to $\uparrow \Delta \%$ hipcidin over menstrual cycle. Higher level of estradiol in MLP may mediate direct or indirect negative feedback on hipcidin synthesis via a physiological regulation or via reduced inflammatory cytokines to increase serum iron levels. Of note, inflammatory cytokines enhance hepcidin synthesis $[15,27,28]$. Collectively, we supposed that higher estrogen may potentially reduce hepcidin synthesis in the subsequent menstrual phase to allow more iron absorption. In accordance to our results, cellular and human clinical studies, e.g., in hepatocyte and among females who administrated progesterone during in-vitro fertilization, reported positive effect of progesterone on hepcidin biosynthesis via binding to membrane-bound progesterone receptor [12]. Increased hepcidin is recorded in high-progesterone states as in early pregnancy [29]. Consequently, increased hepcidin during high progesterone level during MLP in our study matched to this issue. However, these associations failed to explained higher hepcidin level in postMS with reduced female sex hormone levels.

Of note, serum ferritin and iron were the strongest predictors of hipcidin in preMS and postMS. So, hepcidin as the main physiological iron regulatory feedback is preserved. Menses cessation among postmenopausal women leads to higher ferritin. The latter stimulates hepcidin synthesis as a counterbalance mechanism to limit intestinal iron absorption [1].

Regardless of menopausal state or reproductive cycle phase, MetS was associated with higher hepcidin, ferritin, insulin, and HOMA-IR and lower serum iron and unchanged hepcidin/ferritin ratio in our study. We demonstrated cross-talk of MetS with hepcidin. The serum hepcidin level was increased linearly with ascending numbers of classical MetS features, and advance in hepcidin quartiles was associated with increased proportion of MetS among either preMS or postMS. This cross talk was parallel to the previously reported pattern for both serum hipcidin and serum ferritin levels in the population studies [16, 30]. Moreover, we were the first to identify cut off values of hepcidin $\geq 5.8$ and $\geq 10.3 \mathrm{ng} / \mathrm{ml}$ for diagnosis of MetS in preMS and postMS respectively with sensitivity of $93.3 \%$ and $100 \%$ and specificity of $76.5 \%$ and $71.4 \%$ respectively. The low-grade inflammatory state in MetS may be underling cause for elevated hepcidin as inflammatory cytokines were reported to increase hepcidin synthesis [15, 24].

Our study may partly try to answer this issue, why hepcidin was elevated in MetS, postMS and during MLP in preMS via explore the association of hepcidin with insulin, IR, and adipose tissue.

While the relationship between iron stores and IR is bidirectional [13], the association of hepcidin with insulin and IR remains vague and was not addressed before in preMS and postMS. In our study, higher hepcidin level in higher insulin and IR states namely MetS, MLP, and postMS; positive association of hepcidin with insulin and HOMA-IR in EFP and PMS; and identification of insulin as independent predictor of hepcidin in PMS highlighted important regulatory role of insulin on hepcidin particularly in postMS. In this context, insulin stimulates hepcidin synthesis via signal transducer and activator of transcription 3 protein syntheses (STAT3). Moreover, both insulin and hepcidin are localized in pancreatic $\beta$ cell granules and there is concomitant increase of both in glucose-treated subjects [31-33]. Variability and fluctuations of insulin level and HOMA-IR during reproductive cycle may be the causes of not being contributors to hepcidin level in preMS. Also, we found percent increase of serum hepcidin was inversely correlated to percentage increase of insulin and HOMA-IR during reproductive cycle. We supposed that physiological process may mediate negative regulatory feedback of insulin on hepcidin as high insulin level in second half of the cycle leads to decrease hepcidin level to allow iron absorption during menses of subsequent menstrual cycle. However, the association of hepcidin with IR states varies in different population studies. Circulating hepcidin levels are positively correlated with obesity in women but not with the presence of non-alcoholic fatty liver disease. Contrarily, researchers demonstrated either unchanged or low hepcidin synthesis in non-alcoholic fatty liver disease [18, 20, 22]. Hepcidin concentrations vary in different populations with T2D as increased or reduced or unchanged levels were recorded [19].

Another factor was assessed in our study; the role of abdominal SCF and VPF as adipose tissue expresses hepcidin gene and protein [21]. In our study, MetS and postMS were associated with increased abdominal SCF and PVF. In preMS, only abdominal SCF was positively correlated and independent predictors for hepcidin level. Although both abdominal SCF and visceral fat were positively correlated to hepcidin level in postMS, none of them was an independent predictor of hepcidin 
level. Bekri et al. 2006 reported that expression of hepcidin gene and protein in adipose tissue was higher among obese premenopausal women and was positively correlated to inflammation not to iron parameters. They suggested that subcutaneous adipose tissue mass in obese subjects may be accountable for elevated hepcidin level [21]. However, other researchers suggested that adipose tissue may really minimally participate to circulating hepcidin levels as expression of hepatic hepcidin mRNA was $\sim 700$-fold greater than that of adipose tissue and suggesting that hepcidin may have tissue-specific regulation and inflammatory parameters rather than adipose tissue mass may mediate enhanced hepcidin level [22].

According to previous data in literature, higher H/F ratio may indicate either hepcidin resistance or elevated hepcidin level independent of the ferritin level [13-15]. In our study, firstly, H/F did not discriminate between metabolic and non-metabolic groups of the same menopausal state or reproductive cycle phase. This may denote that MetS was not associated with hepcidin resistance but associated with proportionate increase in hepcidin to ferritin; secondly, postMS was associated with higher hepcidin/ferritin ratio than preMS. In details, postMS had higher ratio compared to both the EFP and MLP among MetS subgroups; and EFP in non-MetS subgroups. This was associated with insignificant difference of $\mathrm{H} / \mathrm{F}$ ratio over reproductive cycle in MetS subgroups in spite of higher $\mathrm{H} / \mathrm{F}$ ratio in MLP than EFP in nonMetS subgroups. Another parallel finding is that insulin and HOMA-IR increased during MLP regardless of MetS along with insignificant and significant higher insulin and HOMA-IR in MLP than postMS among MetS and non-MetS subgroups respectively. So, subjects in nonMets subgroups presented marked increased IR parallel to increased H/F ratio during MLP. So, high insulin level among women without MetS may stimulate hepcidin synthesis and lead to higher H/F in MLP. Importantly, researchers demonstrated that insulin resistance was associated with MetS in postMS not in preMS [34]. Further studies are needed to explain this finding.

\section{Conclusion}

Our study was the first to explore the impact of female sex hormones, IR, and MetS on hepcidin level based on menopausal state and reproductive cycle phases. postMS, MetS, and luteal phase of reproductive cycle are independently associated with elevated hepcidin and ferritin levels. Hepcidin may be used as biomarker for diagnosis of MetS. The iron parameters as main hepcidin regulatory factors are preserved in these conditions. We demonstrated that hepcidin regulation differed between preMS and postMS. Abdominal SCF and progesterone were contributors to hepcidin level in the former state, meanwhile insulin,
MetS, and progesterone were contributors in the postMS. Estrogen and progesterone were positively correlated to hepcidin. However, neither estrogen nor progesterone was responsible for higher hipcidin in postMS. H/ F ratio is increased in postMS but unchanged in MetS.

\section{Limitation of our study}

Cross-section study cannot provide cause result relationship; the use of a surrogate measure of insulin resistance by HOMA-IR; we lacked information on levels of testosterone, gonadotropin, and transferrin saturation and genetic mutations that are related to iron disorders.

\section{Abbreviations}

ANOVA: Analysis of variance; AUCs: Areas under curves; CT: Computerized tomography; DH: Dysmetabolic hyperferritinemia; EFP: Early follicular phase; FPG: Fasting plasma glucose; H/F: Hepcidin/ferritin ratio; HDL-C: High-density lipoprotein-cholesterol; HOMA-IR: Homeostatic model for assessment of insulin resistance; IR: Insulin resistance; LDL-.c: Low-density lipoprotein-cholesterol; MetS: Metabolic syndrome; MLP: Mid-luteal phase; postMS: Postmenopausal state; preMS: Premenopausal state; PVF: Peritoneal visceral fat; ROC: Receiver operating characteristic; SCF: Subcutaneous fat thickness.

\section{Acknowledgments}

The authors want to thank all enclosed subjects and Mr. Ahmed Mohamed Abdelraf, master degree of internal medicine.

\section{Authors' contributions}

Dr. Matta provided the ideas and the design of the study and wrote the protocol. She shared in conducting the literature review and data collection, recruited the participants, analysis of the result, and wrote the draft of the manuscript. Prof. Abdelftah followed all stages of the study. Dr. Essawy did the radiological aspect of the study. Dr. Saedii did the laboratory work of this study. And the authors approved the final draft. The author(s) read and approved the manuscript.

\section{Funding}

This study was partially sponsored by Faculty of Medicine, Minia University, Minia, Egypt (pay portion of the chemicals' expenses).

\section{Availability of data and materials}

The datasets generated and analyzed during the present study are not publicly accessible due to concerns of participates confidentiality but are offered by the corresponding author on realistic request.

\section{Declarations}

Ethics approval and consent to participate

Our study was approved by local ethical committee of our institute. All procedures performed in our clinical study including human participants were in accordance with the ethical standards of the institutional and/or national research committee and with the Helsinki Declaration. All individual participants gave informed consent to be included in the study.

\section{Consent for publication}

All the authors involved in this study give their consent for this article to be published in The Egyptian Journal of Internal Medicine. Also, consent for publication was obtained from the each participant in the current study.

\section{Competing interests}

The authors declare that they have no known competing financial interests or personal relationships that could have appeared to influence the work reported in this paper. 


\section{Author details}

${ }^{1}$ Diabetes and Endocrinology Unit, Department of Internal Medicine, Faculty of Medicine, Minia University, Minia, Egypt. ${ }^{2}$ Department of Internal Medicine, Faculty of Medicine, Minia University, Minia, Egypt. ${ }^{3}$ Department of Radiodiagnosis, Faculty of Medicine, Minia University, Minia, Egypt. ${ }^{4}$ Department of Clinical Pathology, Faculty of Medicine, Minia University, Minia, Egypt.

Received: 29 October 2021 Accepted: 26 November 2021

Published online: 21 January 2022

\section{References}

1. Camaschella C, Nai A, Silvestri L (2020) Iron metabolism and iron disorders revisited in the hepcidin era. Haematologica 105(2):260-272

2. Yeung EH, Zhang C, Mumford SL et al (2010) Longitudinal study of insulin resistance and sex hormones over the menstrual cycle: the BioCycle study. J Clin Endocrinol Metab 95(12):5435-5442

3. Angeli A, Lainé $F$, Lavenu $A$ et al (2016) Joint model of iron and hepcidin during menstrual cycle in healthy women. AAPS J 18(2):490-504. https:// doi.org/10.1208/s12248-016-9875-4

4. Jian J, Pelle E, Huang X (2009) Iron and menopause: does increased iron affect the health of postmenopausal women? Antioxid Redox Signal 11(12):2939-2943. https://doi.org/10.1089/ars.2009.2576

5. Galesloot TE, Vermeulen SH, Geurts-Moespot AJ et al (2011) Serum hepcidin: reference ranges and biochemical correlates in the general population. Blood 117(25):e218-e225. https://doi.org/10.1182/blood2011-02-337907 Epub 2011 Apr 28. PMID: 21527524

6. Ali SB, Belfki-Benali H, Aounallah-Skhiri H et al (2014) Menopause and metabolic syndrome in Tunisian women. Biomed Res Int 2014:457131. https://doi.org/10.1155/2014/457131

7. Franklin RM, Ploutz-Snyder L, Kanaley JA (2009) Longitudinal changes in abdominal fat distribution with menopause. Metabolism 58(3):311-315. https://doi.org/10.1016/j.metabol.2008.09.030 PMID: 19217444

8. Hou Y, Zhang S, Wang L et al (2012) Estrogen regulates iron homeostasis through governing hepatic hepcidin expression via an estrogen response element. 511(2):398403. https://doi.org/10.1016/j.gene.2012.09.060 Epub 2012 Oct 3.PMID: 23041085

9. Ikeda Y, Tajima S, Izawa-Ishizawa Y et al (2012) Estrogen regulates hepcidin expression via GPR30-BMP6-dependent signaling in hepatocytes. PLoS One 7(7):e40465. https://doi.org/10.1371/journal.pone.0040465 Epub 2012 Jul 11. PMID: 22792339; PMCID: PMC3394730

10. Yang Q, Jian J, Katz S, Abramson SB, Huang X (2012) 17ß-Estradiol inhibits iron hormone hepcidin through an estrogen responsive element halfsite. Endocrinology 153(7):3170-3178. https://doi.org/10.1210/en.20112045 Epub 2012 Apr 25. PMID: 22535765; PMCID: PMC3380311

11. Lehtihet $M$, Bonde $Y$, Beckman L et al (2016) Circulating hepcidin-25 is reduced by endogenous estrogen in humans. PLoS One 11(2):e0148802. https://doi. org/10.1371/journal.pone.0148802 PMID: 26866603; PMCID: PMC4750915

12. Li X, Rhee DK, Malhotra R, Mayeur C, Hurst LA, Ager (2016) Progesterone receptor membrane component-1 regulates hepcidin biosynthesis. J Clin Invest 126(1):389-401. https://doi.org/10.1172/JCl83831

13. Fernández-Real JM, Manco M (2014) Effects of iron overload on chronic metabolic diseases. Lancet Diabetes Endocrinol 2:513e26

14. Rametta R, Fracanzani AL, Fargion S, Dongiovanni P (2020) Dysmetabolic hyperferritinemia and dysmetabolic iron overload syndrome (dios): two related conditions or different entities? Curr Pharm Des 26(10):1025-1035. https://doi.org/10.2174/1381612826666200131103018 PMID: 32003680

15. Armitage $A E$, Eddowes $L A$, Gileadi $U$ et al (2011) Hepcidin regulation by innate immune and infectious stimuli. Blood 118(15):4129-4139

16. Martinelli N, Traglia M, Campostrini N, Biino G, Corbella M, Sala C (2012) Increased serum hepcidin levels in subjects with the metabolic syndrome: a population study. Edited by Giorgio Sesti. PLoS One 7(10):e48250. https://doi.org/10.1371/journal.pone.0048250

17. Rauber MR, Pilger DA, Cecconello DK, Falcetta FS, Marcondes NA, Faulhaber GAM (2019) Hepcidin is a useful biomarker to evaluate hyperferritinemia associated with metabolic syndrome. An Acad Bras Cienc 91(2):e20180286. https://doi.org/10.1590/0001-3765201920180286 PMID: 31090790

18. Siddique A, Nelson JE, Aouizerat B, Yeh MM, Kowdley KV, Network NCR (2014) Iron deficiency in patients with nonalcoholic fatty liver disease is associated with obesity, female gender, and low serum hepcidin. Clin Gastroenterol Hepatol 12(7):1170-1178
19. Aregbesola A, Voutilainen S, Virtanen JK, Aregbesola A, Tuomainen TP (2015) Serum hepcidin concentrations and type 2 diabetes. World J Diabetes 6(7):978-982. https://doi.org/10.4239/wjd.v6.i7.978

20. Auguet $T$, Aragonès $G$, Berlanga A, Martínez S, Sabench F, Binetti J, Aguilar C, Porras JA, Molina A, Del Castillo D, Richart C (2017) Hepcidin in morbidly obese women with non-alcoholic fatty liver disease. PLoS One 12(10):e0187065. https://doi.org/10.1371/journal.pone.0187065

21. Bekri S, Gual P, Anty R et al (2006) Increased adipose tissue expression of hepcidin in severe obesity is independent from diabetes and NASH. Gastroenterology 131:788-796

22. Tussing-Humphreys LM, Nemeth E, Fantuzzi G et al (2010) Elevated systemic hepcidin and iron depletion in obese premenopausal females. Obesity (Silver Spring) 18(7):1449-1456. https://doi.org/10.1038/oby. 2009.319 Epub 2009 Oct 8. PMID: 19816411

23. Soules MR, Sherman S, Parrott E et al (2001) Executive summary: stages of reproductive aging workshop (STRAW). Fertil Steril 76:874-878

24. Alberti KG, Eckel RH, Grundy SM et al (2009) Harmonizing the metabolic syndrome: a joint interim statement of the International Diabetes Federation Task Force on Epidemiology and Prevention; National Heart, Lung, and Blood Institute; American Heart Association; World Heart Federation; International Atherosclerosis Society; and International Association for the Study of Obesity. Circulation 120:1640-1645

25. Matthews DR, Hosker JP, Rudenski AS, Naylor BA, Treacher DF, Turner RC (1985) Homeostasis model assessment: insulin resistance and beta-cell function from fasting plasma glucose and insulin concentrations in man. Diabetologia 28(7):412-421

26. Rigas AS, Sørensen CI, Pedersen OB et al (2014) Predictors of iron levels in 14,737 Danish blood donors: results from the Danish Blood Donor Study. Transfusion 54(3 Pt 2):789-796

27. Robinson DP, Lorenzo ME, Jian W, Klein SL (2011) Elevated 17b-estradiol protects females from influenza A virus pathogenesis by suppressing inflammatory responses. PLoS Pathol 7:e1002149

28. Hamad M, Awadallah S (2013) Estrogen-dependent changes in serum iron levels as a translator of the adverse effects of estrogen during infection: a conceptual framework. Med Hypotheses 81(6):1130-1134

29. Koenig MD, Tussing-Humphreys L, Day J, Cadwell B, Nemeth E (2014) Hepcidin and iron homeostasis during pregnancy. Nutrients 6(8):30623083. https://doi.org/10.3390/nu6083062

30. Bozzini C, Girelli D, Olivieri O, Martinelli N, Bassi A et al (2005) Prevalence of body iron excess in the metabolic syndrome. Diabetes Care 28:2061-2063

31. Wang H, Li H, Jiang X, Shi W, Shen Z, Li M (2014) Hepcidin is directly regulated by insulin and plays an important role in iron overload in streptozotocin-induced diabetic rats. Diabetes 63:1506-1518

32. Kulaksiz H, Fein E, Redecker P, Stremmel W, Adler G, Cetin Y (2008) Pancreatic beta-cells express hepcidin, an iron-uptake regulatory peptide. J Endocrinol 97(2):241-249

33. Aigner E, Felder TK, Oberkofler $\mathrm{H}$ et al (2013) Glucose acts as a regulator of serum iron by increasing serum hepcidin concentrations. J Nutr Biochem 24:112-117

34. Fonseca JNC, Rocha TPO, Nogueira IAL et al (2018) Metabolic syndrome and insulin resistance by HOMA-IR in menopause. Int J Cardiovasc Sci 31(3):201-208

\section{Publisher's Note}

Springer Nature remains neutral with regard to jurisdictional claims in published maps and institutional affiliations.

\section{Submit your manuscript to a SpringerOpen ${ }^{\circ}$ journal and benefit from:}

- Convenient online submission

- Rigorous peer review

- Open access: articles freely available online

- High visibility within the field

- Retaining the copyright to your article

Submit your next manuscript at $\boldsymbol{s p r i n g e r o p e n . c o m ~}$ 